\title{
Spin densities in pseudoclassical kinetic theory
}

\author{
F. M. C. Witte \\ Institut für Theoretische Physik, Universität Heidelberg, Philosophenweg 19, 69120 Heidelberg, Germany \\ and Julius Institute, Faculty of Physics and Astronomy, University of Utrecht, Leuvenlaan 21, 3584 CE, Utrecht, The Netherlands ${ }^{*}$
}

(Received 8 July 1996; revised manuscript received 13 February 1997)

\begin{abstract}
It is shown that pseudoclassical many-particle systems allow for observable consequences of the spin degrees of freedom, in contrast to the one-particle system, where a consistent formulation of spin space density is impossible. After quantization, this density can then be directly compared to the decomposition of the Wigner operator in terms of spin matrices, in both the nonrelativistic and the relativistic context. From an expansion of the Dirac spinors in terms of large and small components it is seen that in the nonrelativistic limit the pseudoscalar, the timelike component of the axial-vector current, and the spatial components of the vector current vanish. The spatial components of the axial-vector current vanish in the pseudoclassical limit. The pseudoclassical appearance of spin is due to the spin-tensor contribution. [S1050-2947(97)00606-9]

PACS number(s): 03.65.Pm, 03.65.Sq
\end{abstract}

\section{MOTIVATION}

In recent years the prospect of identifying a quark-gluon plasma in nucleus-nucleus or heavy-ion collisions has aroused great interest in the theoretical description of chiral symmetry restoration under nonequilibrium conditions. Much of the effort in understanding the basic physics of this problem has gone into the formulation of a transport theory for the relevant degrees of freedom, quarks, gluons, and mesons. Traditionally these transport theories have been set up for on-shell particles within the framework of a semiclassical approximation. By the methods of field theory similar equations can be derived [1], although the physical interpretation then remains partly obscured due to the difficulty of interpreting the classical limit of quantum field theory in terms of particles. The classical field is dominated by coherent states with an indefinite particle number [2]. One way out is to stick to field theory concepts and to try to go beyond the semiclassical and on-shell approximations [3]. In this paper we rather do the reverse.

The details of the transition from classical to quantum physics, apart from its formal mathematical context, are in general not well understood [4]. In particular, there seems to be no classical analog of the quantum-mechanical spin of fundamental fermions, like quarks and leptons. This should be contrasted with the orbital angular momentum and even the spin 1 of vector bosons, which, apart from their quantized nature, can be accounted for classically. Although this is an intriguing problem by itself, it will not be of concern here. Instead we will focus on a closely related question.

In transport theories for relativistic fermions the classical limit appears to contain a spin density [5]. As spin is considered to be a purely quantum-mechanical effect this state of affairs is at least confusing. The goal of this paper is to analyze this "classical" appearance of spin in detail. We will take the approach of introducing anticommuting coordinates in order to obtain a pseudoclassical description of a

\footnotetext{
*Present address.
}

particle with spin $\frac{1}{2}$. The kinetic theory derived from the underlying pseudoclassical system no longer contains the anticommuting coordinates due to the statistical averaging inherent to any kinetic theory. Within the framework of this paper we identify this resulting kinetic theory, containing only commuting quantities with the physically meaningful one. We hope that a proper treatment of spin eventually improves our understanding of the $\hbar \rightarrow 0$ limit of relativistic quantum transport theories for fermions.

In this paper we treat two questions, closely related to each other. First of all we will briefly discuss the role of spin in pseudoclassical systems. In Sec. II, we will introduce to this end the formalism of pseudoclassical mechanics related to $N=1$ supersymmetry.

It is well known that classical particles with spin $\frac{1}{2}$ can be described in terms of anticommuting variables and that this spin is unobservable in the one-particle system [6]. This construction can be used to discuss various dynamical properties of such particles [7] and it can be generalized to include spin-1 fields such as Yang-Mills bosons [8]. Similarly, one expects a direct relation between the results presented in this paper, and a world line reformulation of quantum field theory [11] is, although extremely interesting, not discussed here. In Sec. III, we will reconsider the question of measurabillity in a many-particle system and set up a transport theory including the spin degrees of freedom. The physical nature of the anticommuting variables seems questionable. In quantum theory, however, we restrict their physical meaning to the expectation values of their products. Here we similarly overcome this problem within a many-particle context.

In Sec. IV we will disentangle the nonrelativistic and classical limiting procedures in the quantum-mechanical appearance of spin. We discuss the spinor decomposition of quantum Wigner functions in both relativistic and nonrelativistic settings. We argue there is no simple Foldy-Wouthuysen transformation that will yield the nonrelativistic Wigner function when applied to the relativistic free theory. An expansion of the relativistic Wigner function in terms of large and small components of the Dirac spinors allows an analysis of the nonrelativistic limit. It can be seen that in the 
classical limit the axial-vector contribution vanishes and the spin-tensor contributions survive. Pseudoscalar contributions vanish due to the nonrelativistic limit. Finally, we summarize the main conclusions.

\section{II. $N=1$ PSEUDOCLASSICAL MECHANICS}

The purpose of this section is to introduce the main tool used in this analysis: $N=1$ pseudoclassical mechanics. We will start out by assigning both commuting and anticommuting coordinates to a single particle. Next we write down a supersymmetric action principle that yields the free-particle equations of motion for the commuting coordinates. After explicitly demonstrating that a particular bilinear form of the anticommuting coordinates represents an intrinsic angular momentum, we will show that it is unobservable. Since this section deals with a rather well documented system, our presentation will resemble earlier works [6].

Let us introduce a supertime variable consisting of a pair $(t, \tau)$ of which $t$ is commuting and $\tau$ satisfies $\tau^{2}=0$. A particle's position is specified by a commuting three-vector $\vec{X}(t, \tau)$, which has the decomposition

$$
\vec{X}(t, \tau)=\vec{x}(t)+\vec{\theta}(t) \tau
$$

because a Taylor expansion in $\tau$ truncates after the first order. Since $\vec{X}$ is a commuting object, so is $\vec{x}$, whereas $\vec{\theta}$ must be anticommuting. A small translation in $(t, \tau)$ space has the following effect on $\vec{X}$ :

$$
\vec{X}(t+\epsilon \tau, \tau+\epsilon)=\vec{X}(t, \tau)+\delta \frac{\partial}{\partial t} \vec{X}(t, \tau)+\epsilon \vec{\theta}(t),
$$

where $\epsilon$ is an infinitesimal anticommuting parameter.

The generators of these "supertranslations" are

$$
\mathcal{Q}=\tau \frac{\partial}{\partial t}-\frac{\partial}{\partial \tau}, \quad H=\frac{\partial}{\partial t},
$$

and they span the algebra

$$
[\mathcal{Q}, H]=[H, H]=0, \quad[\mathcal{Q}, \mathcal{Q}]=H .
$$

The square brackets in this equation are supercommutators, i.e., they are commutators (also denoted $[A, B]_{-}$) when at least one of the entries is commuting and they are anticommutators $\left([A, B]_{+}\right)$when both entries are anticommuting. The transformation rules for the components of $\vec{X}$ are given by

$$
\delta \vec{x}=\alpha \vec{\theta}, \quad \delta \vec{\theta}=\alpha \vec{x}
$$

where $\alpha$ is infinitesimal. If $\mathcal{P}$ is the parity transpormation then we will assume that $\vec{\theta}$ transforms like a vector under parity, i.e., $\mathcal{P}(\vec{\theta})=-\vec{\theta}$. The reason for doing so is obvious from Eq. (2.5). The supersymmetry transformation mixes $\vec{\theta}$ and $\vec{x}$ and so giving them different parity would lead to vector and axial-vector component mixing. This is undesirable. The quantity

$$
\theta_{4}=\frac{1}{6} \epsilon_{a b c} \theta_{a} \theta_{b} \theta_{c}
$$

is a pseudoscalar under the integration measure to be defined later.

If we want to construct a supersymmetric action functional, we need to use derivatives $D$ that are covariant with respect to these translations, that is, $D$ must satisfy

$$
[\mathcal{Q}, D]_{+}=0 \text {. }
$$

It is straightforward to check that

$$
D=\tau \frac{\partial}{\partial t}+\frac{\partial}{\partial \tau}
$$

satisfies this requirement.

\section{A. Free particles}

The general form of a supersymmetric action functional is

$$
S[\vec{X}]=\int d t d \tau \mathcal{L}(\vec{X}),
$$

where the integration is standard over the "normal" time $t$ and Berezin integration over the anticommuting variable $\tau$. Due to the fact that the integration measure $d t d \tau$ is anticommuting, the minimal number of $D$ 's in a nontrivial Lagrangian is 3 . The simplest nontrivial choice therefore is

$$
S_{1}[\vec{X}(t, \tau)]=\int d t d \tau \frac{1}{2} D X_{a} D\left(D X_{a}\right)
$$

where we introduced the latin indices $a=1,2,3$. By working out the two factors

$$
D X_{a}(t, \tau)=-\theta_{a}(t)+\tau \dot{x}_{a}(t)
$$

and

$$
D D X_{a}=\dot{\theta}_{a}(t) \tau+\dot{x}_{a}(t)
$$

one finds after performing the Berezin integration over $\tau$

$$
S_{1}=\int d t \frac{1}{2}\left\{\dot{x}_{a} \dot{x}_{a}-\theta_{a} \dot{\theta}_{a}\right\}
$$

The equations that follow from extremizing this action are

$$
\begin{aligned}
& \ddot{x}_{a}=0, \\
& \dot{\theta}_{a}=0,
\end{aligned}
$$

indeed, for $\vec{x}$, the free-particle equations of motion. 


\section{B. Interaction with an external field}

In order to obtain insight in the physical content of the anticommuting variables $\vec{\theta}$ we add the following interaction term to the action:

$$
V=\frac{1}{2} \epsilon_{a b c} B_{a} \theta_{b} \theta_{c} \tau, \quad B=\text { const. }
$$

This interaction term breaks supersymmetry; it leaves the equation for $\vec{x}$ unaffected, while for the $\vec{\theta}$ we find

$$
\dot{\theta}_{a}=\epsilon_{a b c} B_{b} \theta_{c}
$$

The solution to Eq. (2.16) represents a vector precessing around the $\vec{B}$ axis with a frequency $|B|$. Of course this looks like the precession of an angular momentum in a homogeneous magnetic field. To identify this angular momentum in detail we allow $\vec{B}$ to be $\vec{x}$ dependent. In this case the equation for $\ddot{x}_{a}$ is modified to read

$$
\ddot{x}_{a}=\nabla_{a} B_{b} S_{b},
$$

where we defined the vector $\vec{S}$ by

$$
S_{a}=\frac{1}{2} \epsilon_{a b c} \theta_{b} \theta_{c}
$$

Obviously, $\vec{S}$ acts as an effective magnetic dipole in the $\vec{B}$ field. For $\vec{S}$ to be a proper angular momentum it must satisfy the $\mathrm{SO}(3)$ commutation relations. To check this we insert the expression for $\vec{S}$ into the supersymmetric Poisson brackets defined as

$$
\left[f\left(\theta_{a}\right), g\left(\theta_{b}\right)\right]=\left(\frac{\partial}{\partial \theta_{a R}} f(\theta)\right)\left(\frac{\partial}{\partial \theta_{a L}} g(\theta)\right),
$$

where the subscripts $L$ and $R$ denote left and right derivatives, respectively. The vector defined in Eq. (2.18) indeed satisfies the $\mathrm{SO}(3)$ commutation relations under Poisson bracketing

$$
\left[S_{a}, S_{b}\right]=\epsilon_{a b c} S_{c},
$$

identifying it as an angular momentum. Using Eq. (2.18) we obtain for the time derivative of the spin vector

$$
\dot{S}_{a}=\epsilon_{a b c} \dot{\theta}_{b} \theta_{c}
$$

When we substitute Eq. (2.16) in Eq. (2.21), and use the fact that $\theta^{2}=0$, we find the equation of motion for $\vec{S}$ to read

$$
\dot{S}_{a}=\epsilon_{a b c} B_{b} S_{c}
$$

We can complete the algebra by noting that

$$
\left[\theta_{a}, \theta_{b}\right]=\delta_{a b}
$$

and

$$
\left[\theta_{a}, S_{b}\right]=\epsilon_{a b c} \theta_{c}
$$

So we conclude that the pseudoclassical particle carries an intrinsic angular momentum, i.e., spin. In particular, one can see from its definition (2.18) that spin is an axial vector, i.e., under the parity operation $\mathcal{P}$ we have for the vectors $\vec{x}, \vec{\theta}$, and $\vec{S}$ that

$$
\mathcal{P}\{\vec{x}, \vec{\theta}, \vec{S}\}=\{-\vec{x},-\vec{\theta}, \vec{S}\}
$$

Note that this fixes $\vec{B}$ as an axial vector as well. A consistent formulation of the quantum theory of these anticommuting quantities will indeed force us to assume the spin to be spin $\frac{1}{2}$.

\section{Measurement on anticommuting quantities}

Since all measurements yield real numbers, the existence of an experiment that measures some effect of the anticommuting degrees of freedom is closely linked to the existence of a map $\mathcal{F}$ that maps $\vec{\theta}$ onto the real numbers. In practice, this boils down to some kind of averaging over the anticommuting degrees of freedom

$$
\mathcal{F}:\langle g\rangle=\int d^{3} \theta g(\vec{\theta}) f(\vec{\theta}, t)
$$

with some weight function $f$. Our conventions imply that $\int d^{3} \theta \theta_{1} \theta_{2} \theta_{3}=1$ as a scalar under parity transformations. This choice may deviate from standard notations, but we believe it is more natural. Upon quantization Berezin integration goes over into the Tr operation of taking traces. The latter is obviously a scalar under parity. Consequently, $\left\langle\theta_{4}\right\rangle=\int d^{3} x d^{3} \theta \theta_{4}$ is a pseudoscalar. For the sake of consistency, only commuting objects should generate a nonvanishing average. This constraint on $f$ implies it is of the form

$$
f\left(\theta_{a}\right)=\theta_{1} \theta_{2} \theta_{3}+\frac{1}{2} C_{a} \theta_{a}
$$

The first term here allows pure $c$ numbers to be equal to their average. We must distinguish between the unit 1 and the pseudoscalar unit $\theta_{4}$, i.e.,

$$
\begin{gathered}
\int d^{3} \theta \theta_{1} \theta_{2} \theta_{3}=1, \\
\int d^{3} \theta \frac{1}{6} \epsilon_{a b c} \theta_{a} \theta_{b} \theta_{c}=\left\langle\theta_{4}\right\rangle .
\end{gathered}
$$

The second term in Eq. (2.26) yields an average value for the spin vector $\vec{S}$ by

$$
\left\langle S_{a}\right\rangle=\int d^{3} \theta \epsilon_{a b c} \theta_{b} \theta_{c} C_{d} \theta_{d}=C_{a}\left\langle\theta_{4}\right\rangle .
$$

Obviously, $\vec{C}$ must be a vector since $\vec{S}$ is an axial vector. Since we have chosen $\vec{\theta}$ to be a vector in the introduction to this section, we see that $f$ is a scalar operator.

An additional requirement is [6]

$$
\langle g(\vec{\theta}) g *(\vec{\theta})\rangle \geqslant 0
$$


Yet by inserting the functions

$$
g_{ \pm}\left(\theta_{a}\right)=\theta_{1} \pm \imath \theta_{2},
$$

we obtain

$$
\left\langle g_{ \pm} g_{ \pm}^{*}\right\rangle=\mp 2 C_{3} .
$$

Depending on the sign of $C_{3}$, Eq. (2.31) fails either for $g_{+}$or for $g_{-}$. Choosing $\vec{C}=0$ entirely trivializes $f\left(\theta_{a}\right)$, so we must conclude that in the one-particle system no experiment, i.e., nontrivial $f(\vec{\theta})$, can detect the presence of the anticommuting degrees of freedom. In particular this means, by using Eq. (2.27) with $\vec{C}=0$ in Eq. (2.17), that

$$
\left\langle\ddot{x}_{a}\right\rangle=0 .
$$

The interaction of $\vec{B}$ with $\vec{S}$ causes no observable effect on the equations of motion of the particle.

This may at first sight seem an unavoidable consequence of including degrees of freedom that have an "unphysical" anticommuting nature. Yet upon quantization it can be shown that Eq. (2.31) can be fulfilled [6]. We will postpone the discussion of this to Sec. IV, where we treat a quantized system. In the next section we set out to show that the consequences of Eq. (2.34) can be avoided. Many-particle systems will allow for observable consequences of spin.

\section{III. $N=1$ MANY-PARTICLE SYSTEM}

In this section we will investigate the properties of the spin vector $\vec{S}$ defined in the preceding section, in a manyparticle system. In the case of orbital angular momentum the limit $\hbar \rightarrow 0$ implies that only large quantum numbers will survive. In the case of spin this is obviously no remedy. Yet, from physical experience we know that systems containing an extremely large number of spins allow for observable consequences of the interactions among the spins. So it seems natural to consider a many-particle system as a possible way to study a "classical" appearance of spin. Furthermore, as we will now show, in a two-particle system one can satisfy Eq. (2.31).

\section{A. The two-particle system}

The main problem with the density $f$ in the one-particle system was its inabillity to handle complex functions of the $\theta_{a}$ correctly. A two-particle system offers the opportunity to evade this problem at the expense of restricting the possible values for the total spin. Consider a distribution function of the form

$$
f\left(\vec{\theta}^{1}, \vec{\theta}^{2}\right)=\theta_{1}^{1} \theta_{2}^{1} \theta_{3}^{1} \theta_{1}^{2} \theta_{2}^{2} \theta_{3}^{2}+C_{a} \theta_{a}^{1} \theta_{1}^{2} \theta_{2}^{2} \theta_{3}^{2}+D_{a} \theta_{a}^{2} \theta_{1}^{1} \theta_{2}^{1} \theta_{3}^{1} .
$$

When taking the average

$$
\left\langle g\left(\vec{\theta}^{1}\right) g^{*}\left(\vec{\theta}^{1}\right)+g\left(\vec{\theta}^{2}\right) g^{*}\left(\vec{\theta}^{2}\right)\right\rangle=\mp\left\{C_{3}+D_{3}\right\}
$$

we see that Eq. (2.31) can be satisfied if the total spin vanishes and if we restrict our attention to averages. We are thus lead to the formulation of measurability in a statistical sense. In particular, by using the delta functions

$$
\begin{gathered}
\int \delta\left(\theta-\theta^{i}\right) d \theta=1, \quad \int \theta \delta\left(\theta-\theta^{i}\right) d \theta=\theta^{i}, \\
\delta^{3}\left(\vec{\theta}-\vec{\theta}^{i}\right)=\delta\left(\theta_{1}-\theta_{1}^{i}\right) \delta\left(\theta_{2}-\theta_{2}^{i}\right) \delta\left(\theta_{3}-\theta_{3}^{i}\right)
\end{gathered}
$$

we can rewrite Eq. (3.1) as a two-particle spin space density $F_{2}$,

$$
F_{2}(\vec{\theta})=\prod_{i=2}^{2} \delta^{3}\left(\vec{\theta}-\vec{\theta}^{i}\right)+\sum_{i=1}^{2} C_{a}^{i} \theta_{a} \delta^{3}\left(\vec{\theta}-\vec{\theta}^{i}\right) \prod_{j \neq i} \theta_{1}^{j} \theta_{2}^{j} \theta_{3}^{j},
$$

and averages are calculated from

$$
\langle g(\vec{\theta})\rangle=\int d^{3} \theta\left\{\int \prod_{i} d^{3} \theta^{i} g(\vec{\theta}) F_{2}(\vec{\theta})\right\} .
$$

These averages now behave well. A direct consequence of this reformulation is that the equations of motion for $\vec{x}$ become nontrivial

$$
\left\langle\ddot{x}_{a}^{i}\right\rangle=-\left\langle\nabla_{a} B_{b} S_{b}^{i}\right\rangle, \quad i=1,2 .
$$

We have achieved that the averaging procedure is now well behaved with respect to linear complex functions of $\vec{\theta}$. Furthermore, we notice that the anticommuting variables enter the equations of motion for $\vec{x}$ only through their quadratic combination in $\vec{S}$. In Sec. III B we will see how this can be exploited.

The basic idea is to generalize to an $N$-particle system $F_{N}$, include the commuting dergrees of freedom, and show that the density obtained in this way satisfies a Klimontovich equation. It can then be identified with an exact phase-spin space density by reexpressing all the dependence on $\vec{\theta}$ in terms of the spin $\vec{S}$. Suitably averaging this exact $\mathrm{N}$-particle density then yields a kinetic equation for the averaged phase-spin space density.

\section{B. The pseudoclassical many-particle system}

The purpose of this subsection is to show that for a pseudoclassical $N$-particle system an exact phase-spin space density satisfying a Liouville-type evolution equation can be constructed. The $N$-particle generalization of Eq. (3.4), including the commuting degrees of freedom, is given by

$$
\begin{aligned}
F_{N}(\vec{x}, \vec{p}, \vec{\theta} ; t)= & \sum_{i=1}^{N} \delta^{3}\left(\vec{x}-\vec{x}^{i}(t)\right) \delta^{3}\left(\vec{p}-\vec{p}^{i}(t)\right) \\
& \times\left\{\delta^{3}\left(\vec{\theta}-\vec{\theta}^{i}\right)+C_{a}^{i}(t) \theta_{a} \delta^{3}\left(\vec{\theta}-\vec{\theta}^{i}\right)\right\} \\
& \times \prod_{j \neq i} \delta^{3}\left(\vec{\theta}-\vec{\theta}^{j}\right)
\end{aligned}
$$

as a function of the coordinate $\vec{x}$, momentum $\vec{p}, \vec{\theta}$, and time $t$. The time dependence of $F_{N}$ originates from the particle coordinates that depend on time and from the timedependent vectors $\vec{C}^{j}$. In order to fix this time dependence 
we resort to the so-called Klimontovich equation for exact phase-space densities. Let $K$ be the super-Liouville operator defined by

$$
K=\frac{\partial}{\partial t}+\dot{x}_{a} \frac{\partial}{\partial x_{a}}+\dot{p}_{a} \frac{\partial}{\partial p_{a}}+\dot{\theta}_{a} \frac{\partial}{\partial \theta_{a}}
$$

then it is easy to show that $F_{M}$ satisfies the Klimontovich equation

$$
K F_{N}(\vec{x}, \vec{p}, \vec{\theta} ; t)=0 .
$$

Inserting Eq. (3.7) into the Klimontovich equation, (3.9) yields the expression

$$
\begin{aligned}
& \sum_{i=1}^{N} \dot{C}_{a}^{i}(t) \theta_{a} \delta^{3}\left(\vec{\theta}-\vec{\theta}^{i}\right) \prod_{j \neq i} \delta^{3}\left(\vec{\theta}-\vec{\theta}^{j}\right) \\
& =\sum_{i=1}^{M} C_{a}^{i}(t) \dot{\theta}_{a} \delta^{3}\left(\vec{\theta}-\vec{\theta}^{i}\right) \prod_{j \neq i} \delta^{3}\left(\vec{\theta}-\vec{\theta}^{j}\right),
\end{aligned}
$$

from which to solve for $\vec{C}^{j}(t)$. Equating each summand seperately and using Eq. (2.16) gives

$$
\dot{C}_{a}=\epsilon_{a b c} B_{b} C_{c},
$$

the equation of motion for the vectors $\vec{C}^{j}$. It coincides with the equation for the spin vector $\vec{S}$, as expected.

$F_{N}(\vec{x}, \vec{p}, \vec{\theta} ; t)$ is an exact phase-spin space density satisfying an evolution equation (3.9). Yet the dynamics of the system is still rather simple and will not lead to an interesting kinetic equation because the interactions among the particles are missing. Improving this state of affairs will require us to introduce an $\vec{x}$-dependent vector field $\vec{B}$. Furthermore, the vectors $\vec{C}^{j}$ will then also become dependent on the particle position and hence the spin density, i.e., the second term in Eq. (3.7), will become a local quantity. Consequently, we would once more face the problem of satisfying Eq. (2.31), knowing that it will be violated locally anyhow. However, now the situation is different. We are working in a manyparticle enviroment and we will proceed towards a statistical description of the system. In particular, the question of the measurability of the coordinates $\vec{\theta}$ is no longer of interest since it represents microscopic information. The relevant physical observable relating to the anticommuting degrees of freedom has now become the macroscopic expectation value of $\vec{S}$.

\section{Pseudoclassical transport theory}

In this section we want to take the final step in our argument. We will assume some kind of averaging of the exact density $F_{N}$ and show how the resulting smoothed density $f$ satisfies a transport equation. Let us decompose $F_{N}$ into an averaged part and a fluctuation part as

$$
F_{N}(\vec{x}, \vec{p}, \vec{\theta} ; t)=\left\langle F_{N}(\vec{x}, \vec{p}, \vec{\theta} ; t)\right\rangle_{\mathrm{av}}+\delta F_{M}(\vec{x}, \vec{p}, \vec{\theta} ; t)
$$

The exact nature of the averaging is immaterial; one should note, however, that any averaging over the anticommuting $\vec{\theta}^{i}$ will remove all the dependence on these variables due to the nature of Berezin integration. The expectation value of the exact density will only depend on the expectation values of the unit and the spin vector $\vec{S}$. So

$$
f(\vec{x}, \vec{p}, \vec{S} ; t)=\left\langle F_{N}(\vec{x}, \vec{p}, \vec{\theta} ; t)\right\rangle_{\mathrm{av}}
$$

will only depend on the spins, through their $\vec{C}^{j}$, lying within the volume elements of phase space averaged over.

We add particle interactions by relating the vector field $\vec{B}$ with $f$ through the electrodynamics relation

$$
B_{a}(\vec{x}, t)^{\mathrm{av}}=\frac{\mu_{0}}{2 \pi} \int d^{3} x^{\prime} \frac{\epsilon_{a b c} \epsilon_{b d e}\left\{\nabla_{d} M_{e}\left(\vec{x}^{\prime}\right)^{\mathrm{av}}\right\}\left(x_{c}-x_{c}^{\prime}\right)}{\left|\vec{x}-\vec{x}^{\prime}\right|^{3}},
$$

where the magnetization $\vec{M}$ is given by

$$
M_{a}(\vec{x})^{\mathrm{av}}=\int d^{3} \vec{p} d^{3} \vec{\theta} S_{a} f(\vec{x}, \vec{p}, \vec{\theta} ; t) .
$$

From Eq. (3.12) we see that the exact quantities are related to their averages by adding fluctuations

$$
\begin{gathered}
\vec{B}=\vec{B}^{\mathrm{av}}+\delta \vec{B}, \\
\vec{M}=\vec{M}^{\mathrm{av}}+\delta \vec{M} .
\end{gathered}
$$

By observing that

$$
\dot{\theta}_{b} \frac{\partial}{\partial \theta_{b}} S_{a}=\dot{S}_{a},
$$

we may interpret $f(\vec{x}, \vec{p}, \vec{S} ; t)$ as a function of $\vec{S}$ and use

$$
\dot{\theta}_{b} \frac{\partial}{\partial \theta_{b}} f(\vec{x}, \vec{p}, \vec{S} ; t)=\dot{S}_{b} \frac{\partial}{\partial S_{b}} f(\vec{x}, \vec{p}, \vec{S} ; t)
$$

Since the quantity $\vec{S}$ is commuting and appears at most linearly in all expressions we replace it by its $c$-number representation, its expectation value. All reference to the anticommuting variables has disappeared. Averaging the Klimontovich equation (3.9) now yields

$$
\begin{aligned}
\left\{\frac{\partial}{\partial t}\right. & \left.+\dot{x}_{a} \frac{\partial}{\partial x_{a}}-\frac{\partial\left(B_{k}^{\mathrm{av}} M_{k}^{\mathrm{av}}\right)}{\partial x_{a}} \frac{\partial}{\partial p_{a}}+\left(\epsilon_{a b c} B_{b}^{\mathrm{av}} M_{c}^{\mathrm{av}}\right) \frac{\partial}{\partial S_{a}}\right\} \\
& \times f(\vec{x}, \vec{p}, \vec{S} ; t) \\
= & \left\{\frac{\partial\left(\delta B_{k} \delta M_{k}\right)}{\partial x_{a}} \frac{\partial}{\partial p_{a}}+\left(\epsilon_{a b c} \delta B_{b} \delta M_{c}\right) \frac{\partial}{\partial S_{a}}\right\} \\
& \times \delta F(\vec{x}, \vec{p}, \vec{S} ; t) .
\end{aligned}
$$

The collisions term can be extracted from the right-hand side of this equation. If we assume that as $N \rightarrow \infty$ the fluctuations can be neglected and if we remove the explicit notation from the averaged quantities, we find 
$\left\{\frac{\partial}{\partial t}+\dot{x}_{a} \frac{\partial}{\partial x_{a}}-\frac{\partial(\vec{B} \cdot \vec{M})}{\partial x_{a}} \frac{\partial}{\partial p_{a}}+(\vec{B} \wedge \vec{M}) \frac{\partial}{\partial S_{a}}\right\} f(\vec{x}, \vec{p}, \vec{S} ; t)=0$,

the Vlasov equation for the system. Equation (3.20) describes the transport phenomena that take place in this manyparticle system due to mutual spin-spin interactions. The collission term can be retrieved from the Klimontovich equation by giving the right-hand side of Eq. (3.19) a more detailed treatment [1].

\section{QUANTUM SPIN}

In the previous sections we have established a classical kinetic theory explicitly containing spin degrees of freedom. The result of our labor was an equation [Eq. (3.20)] describing the nonequilibrium physics of a system containing a very large number of particles, each carrying a magnetic dipole moment proportional to its spin. In this section we make contact with the relativistic formulation of quantum kinetic theory for particles with spin. We proceed by formulating a decomposition of the phase-spin space density that upon quantization grows into the spinor decomposition of the fermionic Wigner function [5]. The latter is then expanded in terms of large and small components allowing a careful separation between the nonrelativistic and the classical limit.

\section{A. Nonrelativistic spin}

If we write down the most general, internally consistent, expansion of the phase-spin space density in terms of products of $\vec{\theta}$, one finds

$$
f=s \theta_{1} \theta_{2} \theta_{3}+p \theta_{4}+\vec{C} \cdot \vec{\theta} .
$$

Let us discuss the terms not appearing in this expansion. We could have added a term proportional to

$$
T_{a b} \theta_{a} \theta_{b}+\frac{1}{2} t_{a} \epsilon_{a b c} \theta_{b} \theta_{c}
$$

for some vector $\vec{t}$ and some antisymmetric tensor $T_{a b}$. They will generate an expectation value for $\vec{\theta}$. The vector $\vec{t}$ will give an axial vector contribution to $\langle\vec{\theta}\rangle$, but since this is a vector we must have $\vec{t}=0$. The tensor $T_{a b}$ will give a vectorlike contribution and therefore seems to be acceptable. But remember that $\vec{\theta}$ is an anticommuting quantity. So any consistently defined average value would have to satisfy

$$
\left\langle\theta_{1} \theta_{2}\right\rangle=-\left\langle\theta_{2} \theta_{1}\right\rangle .
$$

But if we rewrite this in terms of connected and disconnected contributions we find

$$
\left\langle\theta_{1} \theta_{2}\right\rangle_{\text {con }}+\left\langle\theta_{2} \theta_{1}\right\rangle_{\text {con }}=2\left\langle\theta_{1}\right\rangle\left\langle\theta_{2}\right\rangle .
$$

This equation can only be true for $\langle\vec{\theta}\rangle=0$ and thus $T_{a b}=0$. Finally, an additional linear contribution of the form

$$
\epsilon_{a b c} a_{a b} \theta_{c}
$$

for some antisymmetric tensor $a_{i j}$ will give a vectorlike contribution to the average of $\vec{S}$, which is axial. So $a_{i j}=0$. We see that Eq. (4.1) is in fact the most general expansion we can make.

Now consider the quantization of the one-particle system. Classically, the anticommuting coordinates $\vec{\theta}$ satisfy the Poisson bracket

$$
\left[\theta_{a}, \theta_{b}\right]_{P}=\delta_{a b} .
$$

Quantization now implies that we make the transition to the anticommutator

$$
\left[\theta_{a}, \theta_{b}\right]_{+}=\imath \hbar \delta_{a b} .
$$

By defining

$$
\sigma_{a}=\iota \sqrt{\frac{2}{\iota \hbar}} \theta_{a},
$$

the components of $\vec{\sigma}$ satisfy the anticommutation relations

$$
\left[\sigma_{a}, \sigma_{b}\right]_{+}=2 \delta_{a b},
$$

defining a Clifford algebra. Note that $\vec{\sigma}$ transforms like a vector under parity, hence they are not generators of $\mathrm{SO}(3)$ or one of its representations and cannot be identified with the Pauli spin matrices. We will return to this point shortly. The $\vec{\sigma}$ can be identified with a set of $2 \times 2$ matrices and substituting them into the one-particle spin density [Eq. (2.27)] yields

$$
f(\vec{\theta})=\left(1+\vec{C} \cdot \frac{2 \imath \vec{\sigma}}{\hbar}\right),
$$

within a factor $(\hbar / 2 l)^{3 / 2}$. The integration over the anticommuting variables that yielded averages in the pseudoclassical limit is now replaced by taking traces over the spin indices. In particular, for the spin operator quantization yields

$$
S_{a}=\epsilon_{a b c} \sigma_{b} \sigma_{c}=\frac{1}{2} \epsilon_{a b c}\left[\sigma_{b}, \sigma_{c}\right]_{-},
$$

directly relating it to the commutator of $\sigma$ matrices. We identify the matrices $S_{a}$ with the Pauli matrices $\vec{\tau}$ via

$$
S_{a}=\tau_{a} .
$$

The anticommutation relations of the $\vec{\sigma}$ matrices can be used to show that $\vec{S}$ and thus $\vec{\tau}$ still satisfy the $\mathrm{SO}(3)$ commutation relations. By using that $\left(\sigma_{a}\right)^{2}=1$ for any $a$ and projecting $\vec{S}$ on $\vec{\sigma}$ we find

$$
\sigma_{a}\left\{\sigma_{b} S_{b}\right\}=\sigma_{a} \epsilon_{b c d} \sigma_{b} \sigma_{c} \sigma_{d}=\sigma_{a} \sigma_{4}=\tau_{a},
$$

which clearly displays the correspondence between the $\vec{\sigma}$ and $\vec{\tau}$. The introduction of two sets of matrices may seem clumsy, but consistency demands it. By using Eq. (4.10) once again we get

$$
\langle\vec{S}\rangle=\operatorname{Tr}\{\vec{S} f(\vec{\sigma})\}=\vec{C}\left\langle\sigma_{4}\right\rangle .
$$


The quantum spin-density operator can satisfy our requirement [Eq. (2.31)] provided

$$
|\vec{C}| \leqslant \frac{1}{2} \hbar
$$

the equality holds for a pure state. [9]

We now turn to the Wigner function $W(\vec{x}, \vec{p})$ defined by

$$
\begin{aligned}
W_{\alpha \beta}(\vec{x}, \vec{p} ; t)= & \int d^{4} y\left\langle\psi_{\alpha}^{*}\left(\vec{x}-\frac{\vec{y}}{2}, t\right) \psi_{\beta}\left(\vec{x}+\frac{\vec{y}}{2}, t\right)\right\rangle \\
& \times \exp \left\{\frac{l}{\hbar} \vec{p} \cdot \vec{y}\right\}
\end{aligned}
$$

in terms of the nonrelativistic field operator $\psi_{\alpha}$, explicitly including the spin indices. It is a $2 \times 2$ matrix in spin space and can thus be decomposed in terms of the generators of the algebra of $2 \times 2$ matrices. These generators are

$$
T_{i}=\left\{\delta, \sigma^{4}=\left(\sigma^{1} \sigma^{2} \sigma^{3}\right), \vec{\sigma}, \sigma^{4} \vec{\sigma}\right\} .
$$

We recognize the scalar, pseudoscalar, vector, and pseudovector contributions in, respectively, $T_{1}, T_{2}, T_{3-5}$, and $T_{6-8}$. In this basis we can decompose $W$ as

$$
W_{\alpha \beta}=s \delta+p \sigma^{4}+\vec{V} \cdot \vec{\sigma}+\vec{A} \sigma^{4} \vec{\sigma} .
$$

Although the Wigner function has only four independent components, using an eight-dimensional basis for the expansion does not double this number. The doubling comes from the extra splitting caused by including parity transformations. Any function can be written as a sum of even-parity and odd-parity functions. Through the relation between $\vec{\sigma}$ and the anticommuting coordinates $\vec{\theta}$ given by Eq. (4.10) we can immediately deduce the naive pseudoclassical form of the distribution function $f(\vec{x}, \vec{p}, \vec{\theta} ; t)$,

$$
f=s^{\prime} \theta_{1} \theta_{2} \theta_{3}+p^{\prime} \theta_{4}+\vec{V}^{\prime} \cdot \vec{\theta}+\vec{A}^{\prime} \cdot \theta_{4} \vec{\theta},
$$

where the primes denote that these coefficients are only up to a factor equal to those in Eq. (4.18). Of course, we see that this exactly matches the decomposition found earlier in Eq. (4.1). The axial vector $\vec{A}^{\prime}$ cannot yield a classical observable due to the fact that for every component of the anticommuting $\vec{\theta}$ we have $\theta_{a}^{2}=0$. So the axial vector in the Wigner function decomposition is a purely quantum-mechanical object and should vanish in the classical limit. The generalization of these results to relativistic Wigner operators for fermions will be the goal of Sec. IV B. In particular, we will see that a pseudoscalar contribution vanishes in the nonrelativistic limit, so that we can set $p=p^{\prime}=0$ in the previous equations.

\section{B. Relativistic spin}

In the case of relativistic fermions the above treatment must be modified. In this subsection we will discuss these modifications without going through the whole derivations of the previous sections again. In particular, we will focus on the decomposition of the Wigner operator and we will not discuss relativistic pseudoclassical kinetic equations.

First of all we have to introduce an anticommuting fourvector $\theta^{\nu}$. Together with the standard commuting coordinates $x^{\nu}$ it forms the commuting object

$$
X^{\nu}(\sigma, \tau)=x^{\nu}(\sigma)+\theta^{\nu}(\sigma) \tau,
$$

where the pair $(\sigma, \tau)$ is now a super-world-line parameter. Upon quantization the algebra of the anticommuting coordinates becomes a Clifford algebra and hence we obtain the identification

$$
\theta^{\mu} \rightarrow \gamma^{\mu}
$$

The pseudoclassical $\theta^{\mu}$ can be constructed from the threedimensional coordinates $\vec{\theta}$ in exactly the same way as, in the quantum-mechanical case, the $\gamma^{\mu}$ are constructed from the $\vec{\sigma}$. If we seek a four-dimensional generalization of $\vec{S}$ we find

$$
S_{a}=\epsilon_{a b c d} \theta_{b} \theta_{c} \rightarrow S_{\alpha \beta}=\epsilon_{\alpha \beta \gamma \rho} \theta_{\gamma} \theta_{\rho},
$$

which it generalizes into an antisymmetric four-tensor rather than into an axial four-vector. Written out this gives

$$
S_{\alpha \beta}=\left(\begin{array}{cc}
0 & \vec{S} \\
\vec{S} & \epsilon_{a b c} \theta_{0} \theta_{c}
\end{array}\right) .
$$

This is in contrast to the existing literature, where spin is rather identified with an axial four-vector. A closer look at the relativistic Wigner function and its nonrelativistic limit will reveal the origin of this contradiction.

\section{Foldy-Wouthuysen transformations and a small component expansion}

The nonrelativistic limit of the Dirac equation can be found systematically within the framework of FoldyWouthuysen transformations [10]. Let $\Psi$ be a Dirac spinor given in terms of two two-spinor components $\Psi=(\phi, \chi)$; then we define a unitary transformation

$$
\Psi \rightarrow e^{-\imath Z} \Psi
$$

The matrix $Z$ is now determined by the requirement that the new Hamiltonian $H^{\prime}$,

$$
H^{\prime}=e^{\imath Z} H e^{-\imath Z},
$$

no longer mixes the different two-spinor components. Physically, this implies that particle and antiparticle excitations decouple. Obviously, only for the free theory can we find an exact transformation of this type. In this case $Z$ is of the form

$$
Z=-\imath \vec{\gamma} \cdot \vec{b} \omega
$$

where $\vec{b}$ is a unit vector. For some interacting cases an approximate Foldy-Wouthuysen transformation can be found for low-energy fermions. A standard result from these considerations is that the relative weight of particle and antiparticle excitations is given by 


$$
\chi \propto \frac{p}{m} \phi
$$

where $p$ is a typical momentum [10].

Now consider the general form of the spinor decomposition of the relativistic Wigner function for Dirac fermions

$$
W=\langle\bar{\Psi} \Psi\rangle=\mathcal{F} \delta+\imath \gamma^{5} \mathcal{P}+\mathcal{V}_{\mu} \gamma^{\mu}+\mathcal{A}_{\mu} \gamma^{5} \gamma^{\mu}+\mathcal{S}_{\mu \nu} \sigma^{\mu \nu},
$$

where we have scalar, pseudoscalar, vector, axial vector, and tensor contributions to the Wigner function. If we apply the previous Foldy-Wouthuysen transformation to the Wigner function, we find that it is not sufficient to reduce Eq. (4.28) to its nonrelativistic form. For example, the timelike component of the axial-vector current $\mathcal{A}^{0}$ is easily seen to be invariant

$$
e^{\imath Z} \gamma^{5} \gamma^{0} e^{-\imath Z}=\gamma^{5} \gamma^{0}
$$

However, it is a $(\phi, \chi)$-mixing quantity and should therefore be eliminated from the expressions. The simplest and for our purposes sufficient method of finding the nonrelativistic limit is by explicitly introducing the large and small components. We rewrite the Wigner function in terms of $\phi$ and $\chi$,

$$
W=\left(\begin{array}{cc}
\left\langle\phi^{*} \phi\right\rangle & \left\langle\phi^{*} \chi\right\rangle \\
-\left\langle\chi^{*} \phi\right\rangle & -\left\langle\chi^{*} \chi\right\rangle
\end{array}\right)=\left(\begin{array}{cc}
W_{\phi} & W_{\operatorname{mix}} \\
-W_{\text {mix }}^{*} & -W_{\chi}
\end{array}\right),
$$

or in terms of the block matrices $W_{i}$. These can be explicitly computed in terms of the coefficients appearing in the expansion (4.28) by using the Dirac representation of the $\gamma^{\mu}$ matrices in terms of the generators of the Clifford algebra $\vec{\sigma}$. For the large components we get

$$
W_{\phi}=\left\{\mathcal{F}+\mathcal{V}^{0}\right\} \delta-\mathcal{A}_{i} \sigma^{4} \sigma^{i}+\mathcal{S}_{i j} \epsilon^{i j k} \sigma_{k} .
$$

Note that the scalar density appearing here is the sum of particle density (which is equal to particles plus antiparticles) and fermion-number density (which is equal to particles minus antiparticles), in which the antiparticle contributions cancel out. The pseudoscalar is a $(\phi, \chi)$-mixing quantity and thus is surpressed in the nonrelativistic limit. If we now take the spin operator defined in terms of the matrices $\vec{\sigma}$ and calculate its average with $W_{\phi}$ we find

$$
\left\langle S_{a}\right\rangle=\left[-\mathcal{A}_{i} \operatorname{Tr}\left\{\sigma^{4} \epsilon_{a b c} \sigma_{b} \sigma_{c} \sigma_{i}\right\}+\mathcal{S}_{i j} \epsilon^{i j k} \operatorname{Tr}\left\{\sigma_{k} \epsilon_{a b c} \sigma_{b} \sigma_{c}\right\}\right] .
$$

Note that the first term reduces to

$$
\mathcal{A}_{a} \operatorname{Tr}\left\{\left(\sigma^{4}\right)^{2}\right\}
$$

and the second to

$$
\epsilon_{a i j} \mathcal{S}_{i j} \operatorname{Tr}\left\{\epsilon_{b c d} \sigma_{b} \sigma_{c} \sigma_{d}\right\} .
$$

Now in the classical limit $\left(\sigma^{4}\right)^{2} \rightarrow\left(\theta_{4}\right)^{2}=0$ and hence the axial-vector contribution vanishes. The tensor contribution will survive because it is not surpressed by the commutation relations. The general structure of Eq. (4.32) is like that of the scalar density. We have a sum of different particle and antiparticle contributions in which the antiparticle contributions cancel. In the quantum-mechanical system we may separate between the magnetic dipole represented by $\mathcal{S}$ and the spin density represented by the axial vector. In the classical limit, however, this spin density is destroyed by the commutation relations and only the magnetic moment survives. If antiparticles are not present, i.e., in the nonrelativistic limit, the expectation values for spin and magneticdipole densities are of course proportional and so the breakdown of the Clifford algebras anticommutation relations causes no loss of physical information. Yet in the classical limit nothing prevents us from going to extremely relativistic energies where the appearance of antiparticles makes the spin density and dipole-moment density physically distinct. In this case the pseudoclassical system still does not allow for a difference between the spin density and magnetic-dipole density unless the antiparticles are introduced by hand.

\section{CONCLUSION}

From the above elaborations we draw the following conclusions. In the pseudoclassical limit of a quantum transport theory for spin- $\frac{1}{2}$ fermions spin can make its appearance in the form of a magnetic-dipole density. In pseudoclassical many-particle systems spin can become observable in a welldefined manner. Basically, the most general phase-spin space density will, in the pseudoclassical limit, reduce to a sum of scalar, pseudoscalar, and vector contributions. An axialvector contribution, as is found in the spin decomposition of the (non)relativistic Wigner function will not survive the classical limit. This is due to the impossibility of dynamically generating antiparticles in a pseudoclassical vacuum. A tensor contribution to the Wigner function, as is found in relativistic quantum transport theory, need not vanish as $\hbar \rightarrow 0$.

\section{ACKNOWLEDGMENT}

This work was supported in part by the Deutsche Forschungs Gemeinschaft, Grant No. Hu 233/43.
[1] W. Botermans and R. Malfliet, Phys. Rep. 198, 115 (1990); S. R. de Groot, W. A. van Leeuwen, and Ch. G. van Weert, Relativistic Kinetic Theory (North-Holland, Amsterdam, 1980); P. Danielewicz, Ann. Phys. (N.Y.) 197, 154 (1990); 152, 239 (1984); B. Blättel, V. Koch, and U. Mosel, Rep. Prog. Phys. 50, 63 (1993); Y. Zhang and L. Wilets, Phys. Rev. C 45,
1900 (1992); H.-T. Elze and U. Heinz, Phys. Rep. 183, 81 (1989); S. Mrowczynski and P. Danielewicz, Nucl. Phys. B 342, 345 (1990); E. Calzetta and B. L. Hu, Phys. Rev. D 37, 2878 (1988).

[2] L. G. Yaffe, Rev. Mod. Phys. 54, 407 (1982).

[3] F. M. C. Witte and S. P. Klevansky (unpublished); P. A. Hen- 
ning, Phys. Rep. 253, 236 (1995); E. Wang and U. Heinz, Phys. Rev. D 53, 889 (1996).

[4] B. L. Hu, Physica A 158, 399 (1988).

[5] D. Vasak, M. Gyulassy, and H.-T. Elze, Ann. Phys. (N.Y.) 173, 462 (1987).

[6] P. G. O. Freund, Introduction to Super Symmetry (Cambridge University Press, Cambridge, 1986).

[7] J. W. van Holten, Nucl. Phys. B 356, 3 (1991); R. H. Rietdijk and J. W. van Holten, Class. Quantum Grav. 10, 575 (1993).
[8] C. M. Hull and J.-L. Vazquez-Bello, Nucl. Phys. B 416, 173 (1994).

[9] L. P. Kadanoff and G. Baym, Quantum Statistical Mechanics (Benjamin, New York, 1962).

[10] C. Itzykson and J.-B. Zuber, Quantum Field Theory (McGrawHill, New York, 1980).

[11] Z. Bern and D. A. Kosower, Phys. Lett. B 298, 1669 (1988); M. J. Strassler, Nucl. Phys. B 385, 145 (1992); M. G. Schmidt and C. Schubert, Phys. Lett. B 318, 145 (1993); Phys. Rev. D 53, 2150 (1996). 